\section{Summary}

A trial is described comparing the effects of aspirin and phenylbutazone in the treatment of acute rheumatic fever. So far as was possible alternate cases were treated with each drug, resulting in totals of 41 cases treated with aspirin and 47 with phenylbutazone. Detailed analysis of the two groups showed them to be comparable.

The rusults were assessed clinically by the time taken for relief of joint pain and swelling, and for resolution of fever and tachycardia. Frequent estimations of the E.S.R. were made in all cases, and in 24 patients serial plasma fibrinogen estimations were carried out in addition.

The clinical symptoms were relieved earlier and the E.S.R. showed a more rapid return to normal in the phenylbutazone group. Phenylbutazone also produced a more rapid return of the plasma fibrinogen to normal levels.

Although acute symptoms recurred during treatment in seven of the cases on aspirin, there was no evidence of recrudescence in the phenylbutazone group.
REFERENCES

Ablard, G., Larcan, A., and Welfringer, A. (1957). Un. méd. Can., 86, 932 .

Begg, T. B., Kerr, J. W., and Knowles, B. R. (1962). Brit. med. f., 2,

Chaptal, J., Jean, R., and Campo, C. (1956). Sem. Hôp. Paris, 32, 1175

Colson, J. A., Uters, M., and Haberer, Ch. (1956). Bull. Soc. Méd. milit. franç., 50, 166 .

Currie. J. P. (1952). Lancet, 2, 15.

- Peebles Brown, R. A., and Will, G. (1953). Ann. rheum. Dis., 12, 88.

Fleming, J., and Will, G. (1953). Ibid., 12, 95.

Fletcher, A. A., Dauphinee, J. A., and Ogryzlo, M. A. (1952). f. clin. Invest., 31, 561 .

Hitchens, R. A. N. (1956). Ann. rheum. Dis., 15, 160.

Joint Report by M.R.C. and American Heart Association (1955). Brit.

med. F., 1, 555.

Jones, T. D. (1944). 7. Amer. med. Ass., 126, 481. Wuzell, W. C., Schaffarzick, R. W., Naugler, W. E., Gaudin, G., and Mankle, E. A. (1953). Arch. intern. Med., 92, 646.

Laplane, R., Debray, P., Salbreux, R., and Polonovski, C. (1957). Bull. Soc. Méd. Paris, 73, 302

Leff, S. (1956). Ann. rheum. Dis., 15, 33.

Losner, S., and Volk, B. W. (1956). Amer. f. med. Sci., 232, 276.

Reid J. and Sproull, D. H. (1957). Brit. med. F., 1, 1089.

Stirland, R. M. (1956). Lancet, 1, 672. 241, 207

Will, G. (1958). Rheumatologie, 10, 103.

\title{
Neonatal Myasthenia Gravis
}

\author{
GERALD M. STERN, ${ }^{*}$ M.B., M.R.C.P. ; JUDITH M. HALL, $\dagger$ M.B., M.R.C.P.ED., D.C.H. \\ DEREK C. ROBINSON, $†$ B.M., M.R.C.P.
}

Brit. med. F., 1964, 2, 284-286

Strickroot et al. (1942) described the case of a mother with myasthenia gravis whose newborn child developed signs of generalized weakness on the third day of life and died on the seventh day from respiratory failure. Since then 34 further cases of neonatal myasthenia gravis have been described in the literature. The potential gravity of the disorder, and its possible bearing on the aetiology of myasthenia gravis, justify reporting the clinical and serological features of two further cases.

\section{Case 1}

In 1956, when aged 23, the mother of this case had complained of double vision and drooping of the lids, which responded dramatically to neostigmine. Two years later she developed weakness of the trunk and proximal limb muscles. The weakness fluctuated in severity, but she was able to remain at work while taking up to $300 \mathrm{mg}$. of pyridostigmine daily. In 1960 she began to complain of occasional aching discomfort and swelling in the joints of both hands, which responded to salicylates. Special investigations revealed no abnormality. A radiograph of the chest showed no evidence of thymic enlargement, plasma proteins and E.S.R. were normal, and lupus erythematosus cells were not seen in her peripheral blood.

She was pregnant for the first time in February 1962 and became weaker during the last three months of her pregnancy. She was noted to have a variable asymmetrical ptosis, impaired ocular movements with diplopia, moderate generalized weakness of the trunk and shoulder-girdle muscles, and brisk tendon reflexes. She had swelling of the interphalangeal joints of both hands, moist palms, and ulnar deviation of her right wrist. Normal foetal movements

\footnotetext{
- Department of Neurology, London Hospital. Present address : Department of Neuroiogy, the Royal Victoria Infirmary, Newcastle upon Tyne.

+ Department of Paediatrics, London Hospital.
}

were present throughout her pregnancy, and in the latter part she required $420 \mathrm{mg}$. of pyridostigmine daily.

During labour she had regular strong uterine contractions, but became fatigued in the second stage and was delivered by forceps of a male infant weighing $7 \mathrm{lb} .8 \mathrm{oz}$. $(3,400 \mathrm{~g}$.) on 18 October 1962.

The infant appeared normal at birth, but when 24 hours old had an attack of hiccups, and excessive mucus was aspirated from the pharynx. Five hours later he was found to be hypotonic, with shallow respiratory excursions. His eyes were staring, he had a feeble cry and a drooping lower jaw, and corneal and suck reflexes were absent. Within 10 minutes he responded to $0.25 \mathrm{mg}$. of intramuscular neostigmine and was found to need $0.3 \mathrm{mg}$. intramuscularly three-hourly, it being given with atropine to counteract the muscarine effects of the neostigmine. Despite this regimen his suck reflex remained weak, his lower jaw sagged occasionally, and he required to be tube-fed for the next two weeks. During the first week of life he had several severe cyanotic attacks. Attempts to wean him off neostigmine were made on the 18th, 22nd, 33rd, and 42 nd days, but each occasion was followed by a relapse, with recurrence of his original symptoms and signs, which responded to further neostigmine. Treatment was finally stopped on the 47th day without ill effects, and he subsequently appeared to be a normal infant. He was last seen when 1 year old.

There were no antibodies to normal human muscle in the mother's serum, using an indirect immunofluorescent technique. Complement-fixation tests on the mother's serum and the baby's serum on the third day of life, using human muscle, brain, liver, thyroid, and placenta as antigens, were all negative. The latex test was negative in both mother and child. Antinuclear factor was present in the serum of both.

\section{Case 2}

In 1958, at the age of 23 and during the fourth month of her first pregnancy, this mother developed generalized weakness, so that climbing. stairs and combing her hair became an effort; a few weeks later she complained of diplopia. Her symptoms persisted through- 
out her pregnancy but she was delivered of a baby boy uneventfully. The diagnosis of myasthenia gravis affecting the mother was not yet established and she had received no anticholinesterases during her pregnancy.

The child cried in a feeble fashion, did not suck, and was noted to have an excess of mucus in its pharynx. He was admitted to another hospital on the fourth day of life because of inability to feed and was found to be dehydrated, cold, and inactive. There was no Moro response, but sucking and rooting reflexes were present. He had a mild degree of hypoplasia of the mandible. The infant was tube-fed for 11 days, after which he fed well, cried lustily, and had no further symptoms.

After this delivery there was a transient remission in the mother's symptoms, but in January 1960 she had a recurrence of generalized weakness followed by diplopia and weakness of chewing and swallowing. A diagnosis of myasthenia gravis was made and she was given neostigmine; for the first time she experienced a marked improvement in her condition. Later, despite increasing doses of neostigmine, her condition deteriorated. She was referred to the London Hospital, where thymectomy was performed in February 1961. The thymus weighed $10 \mathrm{~g}$. and contained many germinal centres. There was a gradual improvement in her condition, but she continued to require anticholinesterase drugs.

During her second pregnancy, which began in August 1962, her strength improved, but she continued to take $180 \mathrm{mg}$. of neostigmine and $180 \mathrm{mg}$. of pyridostigmine daily. A rapid but otherwise normal labour thwarted the plan for her delivery in hospital, and a male infant weighing $6 \mathrm{lb} .5 \mathrm{oz}$. $(2,865 \mathrm{~g}$.) was born at home on 5 May 1963. He appeared well until the second day of life, when he was found to be unable to suck, to become cyanosed after swallowing, and to have lost the ability to cry.

On admission to hospital on the fourth day he was hypotonic and lethargic, with a weak cry and a drooping lower jaw. His eyes were open and staring. Corneal, grasp, sucking, and rooting reflexes were absent and his Moro response was poor. He was given $0.25 \mathrm{mg}$. of neostigmine intramuscularly and responded within 15 minutes. His tone and cry improved and a good Moro response was obtained. During the next four days neostigmine 0.1 to $0.2 \mathrm{mg}$. was given intramuscularly half an hour before each four-hourly feed. Despite this, his suck reflex remained poor and tube-feeding was necessary. Large doses of atropine were required to counteract the muscarine effects of neostigmine, and on the eighth day of life treatment was changed to pyridostigmine. Thereafter atropine was no longer required. Pyridostigmine $0.5 \mathrm{mg}$. was given subcutaneously every eight hours, but tube-feeding had to be continued. On the 12th day one dose of pyridostigmine was omitted and the infant had a severe cyanotic and apnoeic attack. The dose was gradually increased to $1 \mathrm{mg}$. of pyridostigmine subcutaneously before each feed. By the 25 th day he could take his whole feed by bottle. After the 27 th day the dose was gradually decreased, being finally discontinued when he was 40 days old without ill effects. He was discharged 10 days later, feeding and behaving normally. Antinuclear factor was absent in this child's serum.

\section{Discussion}

The clinical features of our cases are similar to those previously described. The neonatal disease is different from that seen in adults and affects only a minority of children born of myasthenic mothers.

Characteristically, the affected infant shows generalized muscular weakness and hypotonia, with little spontaneous movement, and has a weak or absent Moro response. The infant's cry is feeble, and feeding and swallowing are impaired. The eyes are wide open and staring, respiratory movements are shallow, and there may be episodic attacks of cyanosis. The onset of weakness may be observed at birth, within a few hours, or even after two or three days. If the child is severely affected death may occur in the first week from respiratory failure, and three fatalities have been recorded. The illness lasts between 10 and 90 days, with an average of about three weeks. No further myasthenic symptoms have been reported in children who have recovered from the neonatal illness.
From the literature it is difficult to be sure of the incidence. Some fatal cases may not have been diagnosed, and some cases are so mildly affected that recovery occurs without the assistance of drugs as is probable in the sibling of our second patient. The recorded cases tend to be severely affected infants. Viets and Brown (1951) found three children with neonatal myasthenia gravis out of 36 children born of myasthenic mothers. Fraser and Turner (1953) found one infant affected among 22 children of 12 myasthenic mothers. In 10 years (1954-63) six myasthenic mothers have given birth to eight children at the London Hospital ; two of these have been affected.

There is no apparent difference in the clinical features of the disease in women whose infants were normal and those whose infants were affected. There is no obvious relation between maternal dose of anticholinesterases and the severity of the neonatal disease. Thymectomy does not prevent the occurrence of neonatal myasthenia gravis, as is borne out in our second case. Myasthenic mothers have had normal births before having affected children, and, although there are several records of two consecutive myasthenic infants, there is no mention of a normal baby born after a myasthenic one.

In all of the recorded cases promptly treated with parenteral anticholinesterases in adequate doses and repeated suction of the upper respiratory tract the patients recovered completely. However, the gravity of the disease is indicated by the severe cyanotic attacks which occurred in both our cases-in the first while the infant was receiving regular treatment, and in the second when one dose of pyridostigmine was omitted.

Although the clinical features of adult myasthenia gravis have been recognized since the latter half of the nineteenth century, neonatal myasthenia gravis was not described until soon after the introduction of anticholinesterase drugs. It has therefore been postulated that during intrauterine life the infant's muscles become adapted to function in the presence of anticholinesterases and that neonatal symptoms are due to the withdrawal of drugs. If this were the complete explanation it would be expected that many more children of myasthenic mothers would be affected. The symptoms of the mother of our second case suggested that she was myasthenic when she gave birth to her first child, who, in retrospect, was probably affected, although no anticholinesterases had been taken during pregnancy. Kibrick (1954) described a very similar case of an infant who also had hypoplasia of the mandible, and who was born after the mother had developed symptoms of myasthenia gravis but before she had been diagnosed or treated. A presumptive diagnosis of neonatal myasthenia was made later, after the birth of a severely affected sibling. It is more likely that maternal medication influences the delay before symptoms are apparent in the infant.

Churchill-Davidson and Wise (1963) have reported the myasthenic-like electrophysiological properties of the muscles of normal newborn infants born of normal mothers, demonstrating a remarkable tolerance to depolarizing agents and characteristic responses to tetanic stimulation. Presumably some of the infants of myasthenic mothers are exposed to additional factors which convert a latent symptomless condition into an overt and potentially fatal clinical disorder.

There has been some evidence (Walker, 1938 ; Wilson and Stoner, 1944), albeit conflicting, that adult myasthenia gravis might be associated with a circulating substance exerting a curare-like effect on neuromuscular function. Stricker et al. (1960) have shown that patients severely affected with myasthenia gravis temporarily improve after haemodialysis. This could be attributed to the removal of such a substance from the patient's blood-stream.

Simpson (1960) and Nastuk et al. (1960) have suggested that myasthenia gravis might be an autoimmune disorder, and 
recently antibodies to human muscle have been detected in the serum of some patients with myasthenia gravis (Strauss et al., 1960 ; Beutner et al., 1962 ; van der Geld et al., 1963). White and Marshall (1962) have also demonstrated antinuclear factor in the serum of six out of 16 patients with myasthenia gravis. A close correlation between these serological observations and clinical manifestations has not yet been described, and it would be difficult to account for the reported improvement after dialysis (Stricker et al., 1960), as it is unlikely that antibodies could be cleared from the circulation.

Some of the clinical features of neonatal myasthenia gravis, particularly its duration, its complete remission, and its tendency to affect consecutive infants, are compatible with the suggestion (Beutner et al., 1962) that the disease might represent the passive transfer of maternal antibodies. The clinical details of our cases could be interpreted in accordance with this suggestion, but the available serological information offers no confirmation of transfer of antibodies. It appears unlikely that muscle antibodies of antinuclear factor can be directly related to the neonatal disorder. In Case 1 complement-fixation tests and an indirect immunofluorescent technique failed to detect any circulating antibodies to normal human muscle. Antinuclear factor may have crossed from the maternal circulation to the foetus in Case 1, but it was absent in the serum of Case 2.

Until further observations, possibly utilizing more sensitive serological methods, correlate the passage of antibodies from mother to child with clinical disturbance in the infant, the view that neonatal myasthenia gravis is the manifestation of passive transfer of maternal antibodies must remain speculative and unproved.

\section{Summary}

Two cases of transient neonatal myasthenia gravis are reported. In the first case circulating antibodies to muscle could not be detected, but antinuclear factor was present in the serum of mother and child. Antinuclear factor was not present in the serum of the second infant.

The disorder may be more common than the literature suggests. The potential gravity of the unrecognized illness and the favourable therapeutic response to adequate doses of parenteral anticholinesterases are stressed. The condition may affect the children of untreated myasthenic mothers. Maternal medication probably influences the delay before symptoms are apparent in the child.

Current views on the nature of myasthenia gravis are reviewed. The available serological information in our cases does not confirm the hypothesis that transient neonatal myasthenia gravis represents the passive transfer of maternal antibodies.

We are grateful to the physicians and surgeons concerned for permission to publish cases under their care, and to the Muscular Dystrophy Group, who aided this work with a grant for secretarial assistance.

\section{REFERENCES}

Beutner, E. H., Witebsky, E., Ricken, D., and Adler, R. H. (1962). F. Amer. med. Ass., 182, 46 .

Churchill-Davidson, H. C., and Wise, R. P. (1963). Anaesthesiology, 24, 271.

Fraser, D., and Turner, J. W. A. (1953). Lancet, 2, 417.

Kibrick, S. (1954). Pediatrics, 14, 365.

Nastuk, W. L., Plescia, O. J., and Osserman, K. E. (1960). Proc. Soc. exp. Biol.' (N.Y.), 105, 177.

Simpson, J. A. (1960). Scot. med. F., 5, 419.

Strauss, A. J., Seegal, B. C., Hsu, K. C., Burkholder, P. M. Nastuk, W. L., and Osserman, K. E. (1960).' Proc. Soc. exp. Biol. (N.Y.), 105, 184.

Stricker, E., Thölen, H., Massini, M. A., and Staub, H. (1960). F. Neurol. Neurosurg. Psychiat., 23, 291.

Strickroot, F. L., Schaeffer, R. L., and Bergo, H. L. (1942). F. Amer. med. Ass., 120, 1207.

van der Geld, H., Feltkamp, T. E. W., van Loghem, J. J., Oosterhuis, H. J. G. H., and Biemond, A. (1963). Lancet, 2, 373.

Viets, H. R., and Brown, M. R. (1951). New Engl. 7. Med., 245, 647.

Walker, M. B. (1938). Proc. roy. Soc. Med., 31, 722.

White, R. G., and Marshall, A. H. E (1962). Lancet, 2, 120

Wilson, A., and Stoner, H. B. (1944). Quart. F. Med., 13, 1.

\footnotetext{
* Associate Professor of Pharmacy and Biopharmaceutics, School of Pharmacy, State University of New York, Buffalo 14, N.Y., U.S.A. † Associate Chief of Staff, Vererans Administration Hospital, Palo Alto, Calif., U.S.A.
}

frequency of the maintenance doses can be determined only if the rate of elimination of the drug is known (Levy, 1963).

Surprisingly, data concerning the kinetics of elimination of salicylates by humans are very sparse, despite the long history and wide use of these drugs. It was thought desirable, therefore, to determine the rate of elimination of salicylate in a group of subjects under conditions which permitted evaluation of both intersubject and intrasubject variations.

\section{Experimental}

Seventeen healthy human subjects, consisting of seven men and ten women, aged 23 to 53 , weighing 52 to $96 \mathrm{~kg}$., were given $1 \mathrm{~g}$. (three 0.33 -g. tablets) of aspirin on an empty stomach. Blood samples were taken two, four, six, eight, and in most cases also 10 hours after drug administration. Plasma salicylate concentrations were determined by the colorimetric method of Trinder (1954). After one week the test was repeated. 